\title{
Lesion Segmentation and Bias Correction in Breast Ultrasound B-mode Images Including Elastography Information
}

\author{
Gerard Pons ${ }^{a}$, Joan Martía, Robert Martía, Mariano Cabezas ${ }^{a}$, Andrew di Battista ${ }^{b}$, and J. \\ Alison Noble ${ }^{b}$ \\ ${ }^{a}$ Department of Computer Architecture and Technology, University of Girona, Girona, Spain \\ ${ }^{b}$ Department of Engineering Science, University of Oxford, Oxford, United Kingdom
}

\begin{abstract}
Breast ultrasound (BUS) imaging is an imaging modality used for the detection and diagnosis of breast lesions and it has become a crucial modality nowadays specially for providing a complementary view when other modalities (i.e. mammography) are not conclusive. However, lesion detection in ultrasound images is still a challenging problem due to the presence of artifacts such as low contrast, speckle, inhomogeneities and shadowing. In order to deal with these problems and improve diagnosis accuracy, radiologists tend to complement ultrasound imaging with elastography data. Following the prominent relevance of elastography in clinical environments, it is reasonable to assume that lesion segmentation methods could also benefit from this complementary information. This paper proposes a novel breast ultrasound lesion segmentation framework for B-mode images including elastography information. A distortion field is estimated to restore the ideal image while simultaneously identifying regions of similar intensity inhomogeneity using a Markov Random Field (MRF) and a maximum a posteriori (MAP) formulation. Bivariate Gaussian distributions are used to model both B-mode and elastography information. This paper compares the fused B-mode and elastography framework with B-mode or elastography alone using different cases, including extreme cases, where B-mode shows a well defined lesion and where elastography provides more meaningful information, showing a significant improvement when B-mode images are not conclusive which is the often the case in non cystic lesions. Results show that combining both B-mode and elastography information in an unique framework makes the algorithm more robust and image quality independent.
\end{abstract}

Keywords: Breast lesion segmentation, Elastography, Ultrasound, Bias correction, Markov Random Fields

\section{INTRODUCTION}

Breast ultrasound elastography is a recent diagnostic technique which is based on imaging the difference in stiffness between cancerous and benign tissues. Clinical studies support the use of this technique to enrich current screening methods. ${ }^{1-3}$ Following the prominent relevance of elastography in clinical environments, it is reasonable to assume that lesion segmentation methods could also benefit from this complementary information. This paper proposes a novel lesion segmentation framework that takes intensity information (B-mode) and elastography information into account using a Markov Random Field (MRF) and a maximum a posteriori (MAP) formulation. Previous work had fused RF signal with elastography ${ }^{4}$ or had used 3D US segmentation to extract elastography information ${ }^{5}$ but, to our knowledge, this is the first attempt to incorporate both B-mode and elastography information in a common framework for breast lesion segmentation. The structure of the paper is as follows, the method is outlined in Section 2 and evaluated in Section 3 using B-mode information alone, elastography alone and the combination of both modalities. Section 4 concludes the paper and discusses future work.

Further author information: Send correspondence to Gerard Pons, gpons@eia.udg.edu. 


\section{METHODOLOGY}

The proposed methodology is based on the work of Xiao et al., ${ }^{6}$ who proposed a breast ultrasound lesion segmentation framework for B-mode images. They simultaneously estimated a distortion field (considered as an additive artifact of the logarithmic ideal image) to restore the ideal image while at the same time identifying regions of similar intensity inhomogeneity with an MRF-MAP framework using exclusively intensity information. This framework can be updated using multivariate distributions to include additional information. In this paper we focus on including elastography information hence a bivariate framework is presented.

\subsection{Image Model}

Elastography information can be seen as a grayscale image. Indeed, in clinical environments elastography information is commonly shown as an overlay in B-mode screening process assigning intensity value ranges to a different color to visually help radiologists (see Figure 1). Hence, elastography information can be combined with B-mode information to improve segmentation results, mostly when B-mode image information is not enough to locate the lesion.

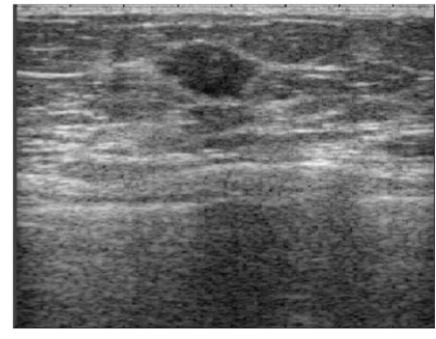

(a)

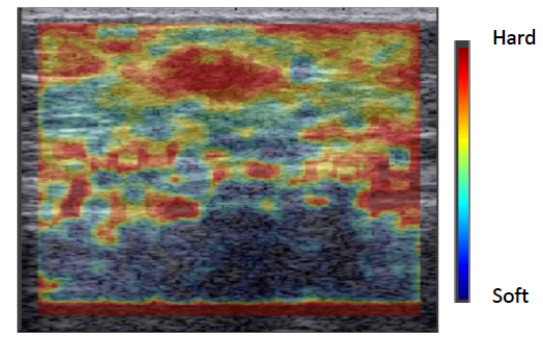

(b)

Figure 1. Figure (a) plots the B-mode image of a DCIS lesion and (b) its elastography information represented by a color overlay. Note that red color represents high values of stiffness (hard tissue) and blue color low values (soft tissue)

The proposed methodology is based on the work of Xiao et al., ${ }^{6}$ who proposed a breast ultrasound lesion segmentation framework for B-mode images. They simultaneously estimated a distortion field (considered as an additive artifact of the logarithmic ideal image) to restore the ideal image while at the same time identifying regions of similar intensity inhomogeneity with an MRF-MAP framework using exclusively intensity information. This framework can be updated using multivariate distributions to include additional information. In this paper we focus on including elastography information hence a bivariate framework is presented.

In this work we assume that both images (B-mode and elastography) present intensity inhomogeneities and these are described by a multiplicative field. A logarithmic transformation yields such multiplicative model to an addition. Let the observed and ideal log-transformed intensities be respectively denote by $\mathbf{y}$ and $\mathbf{y}^{*}$, then $\mathbf{y}=\mathbf{y}^{*}+\mathbf{d}$ where $\mathbf{d}$ denotes the log-transformed intensity bias field. Given the class label $x_{i} \in \mathcal{L}$, where $\mathcal{L}$ denotes the label set. Note that $\mathbf{y}_{i}^{*}$ at pixel $i$ refers to both B-mode and elastography values at that pixel. It is assumed that those values, $\mathbf{y}_{i}^{*}$, follow a bivariate Gaussian distribution with parameter $\theta\left(x_{i}\right)=\left(\boldsymbol{\mu}_{x_{i}}, \Sigma_{x_{i}}\right)$, where $\boldsymbol{\mu}_{x_{i}}$ are both means for B-mode and elastography and $\Sigma_{x_{i}}$ the covariance matrix of class $x_{i}$.

$$
p\left(\boldsymbol{y}_{i}^{*} \mid x_{i}\right)=g\left(\boldsymbol{y}_{i}^{*} ; \theta\left(x_{i}\right)\right)=\frac{1}{2 \pi \operatorname{det}\left(\Sigma_{x_{i}}\right)^{\frac{1}{2}}} \exp \left[\frac{1}{2}\left(\boldsymbol{y}_{i}^{*}-\boldsymbol{\mu}_{x_{i}}\right)^{T} \Sigma_{x_{i}}^{-1}\left(\boldsymbol{y}_{i}^{*}-\boldsymbol{\mu}_{x_{i}}\right)\right]
$$

Experimental results corroborates the validity of the assumption of bivariate Gaussian distribution for both lesion and background information in B-mode and Elastography as shown in Fig. 2

Considering the bias field, the above distribution can be rewritten in terms of the observed intensities $\boldsymbol{y}_{i}$ as

$$
p\left(\boldsymbol{y}_{i} \mid x_{i}, \boldsymbol{d}_{i}\right)=g\left(\boldsymbol{y}_{i}-\boldsymbol{d}_{i} ; \theta\left(x_{i}\right)\right)
$$

and the class-independent intensity distribution is denoted by 


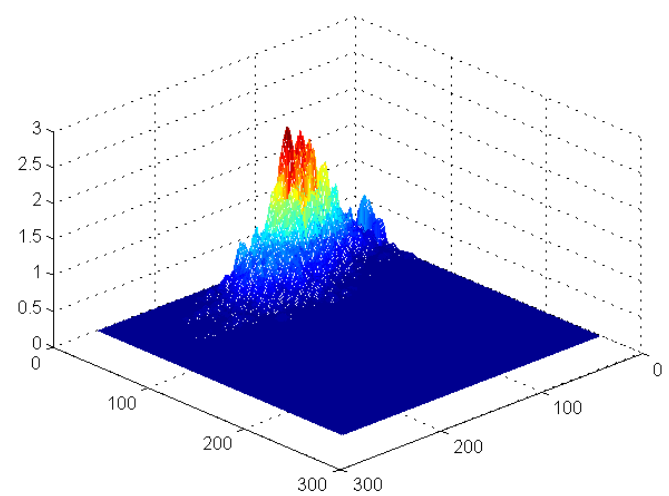

(a)

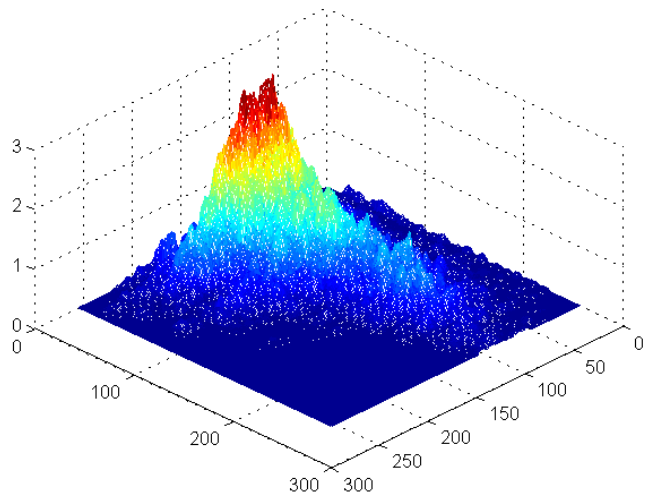

(b)

Figure 2. Examples of bivariate distributions: 2D histograms showing B-mode and Elastography information of (a) lesion and (b) background.

$$
p\left(\boldsymbol{y}_{i} \mid \boldsymbol{d}_{i}\right)=\sum_{j \in \mathcal{L}} g\left(\boldsymbol{y}_{i}-\boldsymbol{d}_{i} ; \theta\left(x_{i}\right)\right) p\left(x_{i}=j\right)
$$

Thus, the intensity distribution is modeled as a bivariate Gaussian mixture and, assuming that the pixel intensities are statistically independent, the probability density for the entire image given the bias field is

$$
p(\boldsymbol{y} \mid \boldsymbol{d})=\prod_{i \in \mathcal{S}} \sum_{j \in \mathcal{L}} g\left(\boldsymbol{y}_{i}-\boldsymbol{d}_{i} ; \theta\left(x_{i}\right)\right) p\left(x_{i}=j\right)
$$

where $\mathcal{S}$ denotes the image pixels set.

\subsection{Label Estimation Using MRF-MAP Framework}

Markov Random Field (MRF) formulation is needed to keep spatial information. It is assumed that neighboring sites have direct relationship with each other and they tend to have the same class labels. The probability density of a MRF model can be described as a Gibbs distribution ${ }^{7}$

$$
p(x)=Z^{-1} \exp [-U(x)]
$$

which defines an energy function as

$$
U(x)=\beta \sum_{c \in \mathcal{C}} V_{c}(x)
$$

where $V_{c}(x)$ is a clique potential over all possible cliques $\mathcal{C}$ which is defined as a subset of pixels in $\mathcal{S}$ where every pair of pixels are neighbors. $\beta$ is a positive constant which controls the size of that clique and $Z$ is a normalization term. The clique potential of $x_{i}$ with respect to its clique neighbor $x_{i}^{\prime}$ is of the form

$$
V_{x_{i}, x_{i}^{\prime}}\left(x_{i}\right)= \begin{cases}0 & \text { if } x_{i}=x_{i}^{\prime} \\ 1 & \text { if otherwise }\end{cases}
$$

Once the energy function $U(x)$ is formulated, the maximum a posteriori (MAP) of class labels is

$$
\hat{x}=\arg \min _{x \in \mathcal{X}} U\left(\boldsymbol{y}^{*} \mid x\right)+U(x)
$$


where $U\left(\boldsymbol{y}^{*} \mid x\right)$ is the likelihood energy given by

$$
U\left(\boldsymbol{y}^{*} \mid x\right)=\sum_{i \in \mathcal{S}}\left[\frac{1}{2}\left(\boldsymbol{y}_{i}^{*}-\boldsymbol{\mu}_{x_{i}}\right)^{T} \Sigma^{-1}\left(\boldsymbol{y}_{i}^{*}-\boldsymbol{\mu}_{x_{i}}\right)+\log \left(\operatorname{det}(\Sigma)^{\frac{1}{2}}\right)\right]
$$

\subsection{Expectation-Maximization (EM) Algorithm}

To obtain the estimation of the distortion field, the EM algorithm is used to update one label image and intensity inhomogeneity field iteratively, initializing the bias field $\boldsymbol{d}$ to be zero. Since a fast convergence is needed in a few iterations an ICM (Iterated Conditional Modes) algorithm ${ }^{8}$ is used.

Expectation step: calculates the posterior tissue class probability $\boldsymbol{W}_{i j}$ using the MRF prior model when the bias field $\boldsymbol{d}$ is known and using $p\left(x_{i}=j \mid x_{\mathcal{N}_{i}}\right)$ which has the form of Eq. 4

$$
\boldsymbol{W}_{i j}=\frac{p\left(x_{i} \mid \boldsymbol{y}_{i}, \boldsymbol{d}_{i}\right) p\left(x_{i}=j \mid x_{\mathcal{N}_{i}}\right)}{p\left(\boldsymbol{y}_{i} \mid \boldsymbol{d}_{i}\right)}
$$

Maximization step: the bias field $\boldsymbol{d}$ is estimated when $\boldsymbol{W}_{i j}$ is known.

$$
\boldsymbol{d}_{i}=\frac{[F \boldsymbol{R}]_{i}}{\left[F \boldsymbol{\psi}^{-1}\right]_{i}}
$$

where $F$ is a low-pass filter and $\boldsymbol{R}$ is the mean residual values for both B-mode and elastography images in which for pixel $i$

$$
\boldsymbol{R}_{i}=\sum_{j \in \mathcal{L}} \boldsymbol{W}_{i j}\left(\boldsymbol{y}_{i}-\boldsymbol{\mu}_{j}\right) \Sigma^{-1}
$$

and $\boldsymbol{\psi}$ is the mean inverse covariance matrix

$$
\boldsymbol{\psi}_{i}^{-1}=\sum_{j \in \mathcal{L}} \boldsymbol{W}_{i j} \Sigma^{-1}
$$

\section{RESULTS}

Although a qualitative evaluation is currently carrying on, a preliminary qualitative evaluation is here presented to show the behavior of the algorithm dealing with extreme cases, one where the lesion is easily detected and segmented using B-mode information alone and one where this modality is clearly insufficient. Firstly, images are segmented using traditional B-mode segmentation. ${ }^{6}$ Subsequently, the image is segmented using the proposed method including both B-mode and elastography information. We have compared the different results in order to determine the benefits to combine both ultrasound information in the lesion segmentation problem. All these experiments have been tested in comparison with manual delineations of the tumors performed by an expert radiologist using B-mode information. Elastography ground truth will be used in the future.

Figure 3 shows the segmentation results for patient 1 . First row of images refers to B-mode segmentation. In this case, B-mode does not provide enough information to detect clearly the tumor and the method fails. Table 1 shows the quantitative results for this patient. Note that Dice Similarity Coefficient (DSC) is lower than 0.3 which indicates the difficulty of the case. In this kind of images elastography provides better information of the tumor location as is shown in second and third row of Fig. 3. Using this information, the DSC is close to 0.7, which indicates a good segmentation result.

On the other hand Fig. 4 shows the segmentation results for patient 2 where the tumor is well defined in the B-mode image and elastography does not provide essential information. Breast US images are not commonly as well defined as this example, but we pretend to compare the algorithm response in extreme cases. Hence, such as we expected, best results are obtained using the traditional framework with B-mode US image alone as 

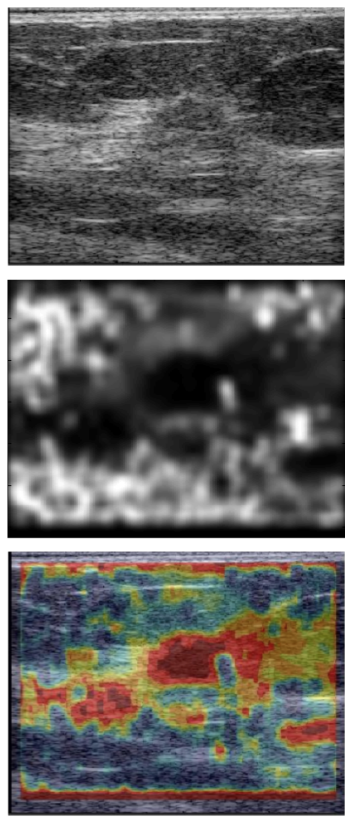

(a)
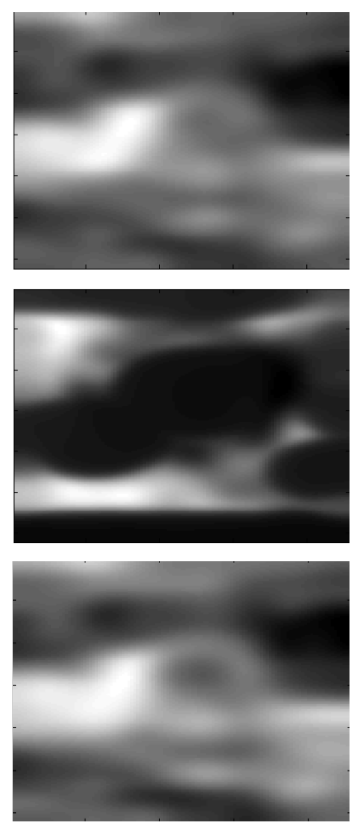

(b)
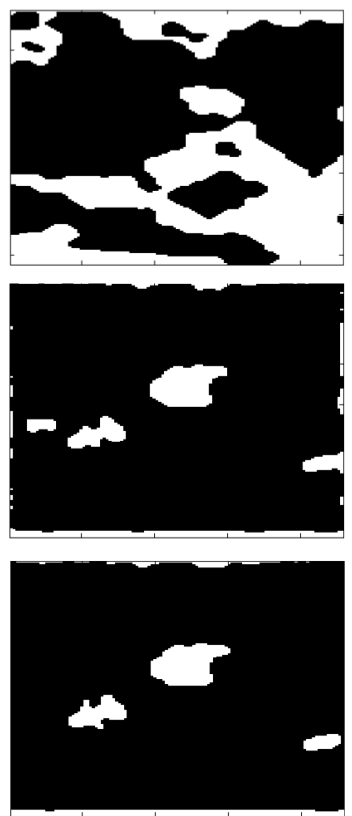

(c)
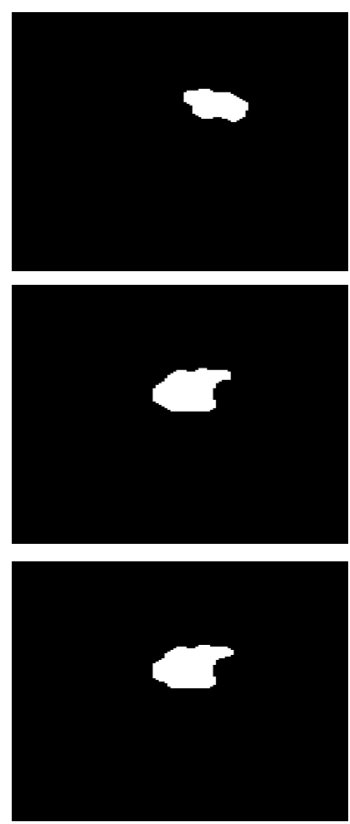

(d)

Figure 3. Segmentation results for Patient 1: using B-mode information alone (first row), using elastography information alone (second row) and combining both modalities (third row). Column (a) plots the original image, (b) the estimated bias field, (c) the first segmentation result and (d) the final refined segmentation.

Table 1. Quantitative segmentation results

\begin{tabular}{|l|l|c|c|c|c|c|}
\hline & Method & Sensitivity & Specificity & PPV & Area Overlap & DSC \\
\hline \hline \multirow{3}{*}{ Patient 1 } & B-mode & 0.3644 & 0.5655 & 0.2404 & 0.1694 & 0.2897 \\
& Elastography & 0.6802 & 0.7783 & 0.7177 & 0.5366 & 0.6984 \\
& Combination & 0.7198 & 0.9906 & 0.6753 & 0.5348 & 0.6969 \\
\hline \hline \multirow{3}{*}{ Patient 2 } & B-mode & 0.9261 & 0.9065 & 0.8971 & 0.8371 & 0.9113 \\
& Elastography & 0.6911 & 0.8411 & 0.8131 & 0.5964 & 0.7472 \\
& Combination & 0.8745 & 0.9929 & 0.8013 & 0.7187 & 0.8363 \\
\hline
\end{tabular}

is shown in Table 1. Graphically, note that elastography information obtains an over-segmented result because tumors invade surrounding tissues making them to appear stiffer. In this case, the method proposed in this paper obtained better results than using elastography information only but slightly worse than using B-mode information alone. However the difference between our proposal and the best method is less than 0.075 when the difference in patient 1 is greater than 0.4 .

\section{CONCLUSION}

This paper has proposed a unified framework for simultaneously estimating the bias field and segmenting lesions in breast ultrasound using both B-mode and elastography information. We have compared our approach with Bmode or elastography alone using two extreme cases, one where B-mode shows a well defined lesion and another where elastography provides more meaningful information. Our proposal has obtained satisfactory results in both cases, showing that combining both B-mode and elastography information in an unique framework makes the algorithm more robust and image quality independent. Results have been improved, especially when B-mode images are not conclusive which is the often the case in non-cystic lesions. However, care has to be taken when selecting the ground truth because elastography images tend to provide larger lesions due to the fact that the surrounding tissue is also stiff. This will be investigated in the future, probably correlating the segmentation results with histological information to assess the extension of the lesion. Additional future work will be focused on exhaustively evaluating the proposed method in a larger data set. 

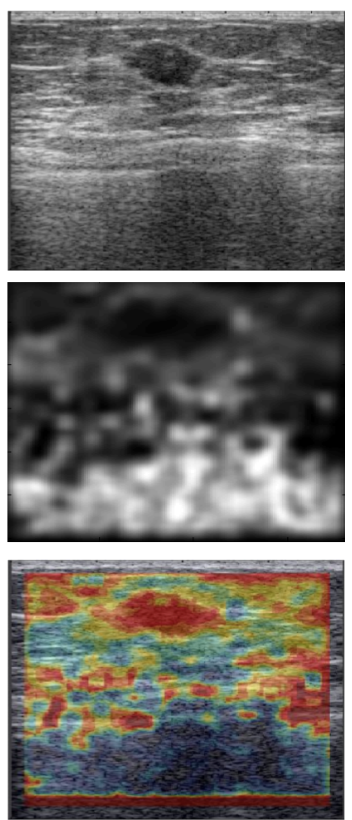

(a)
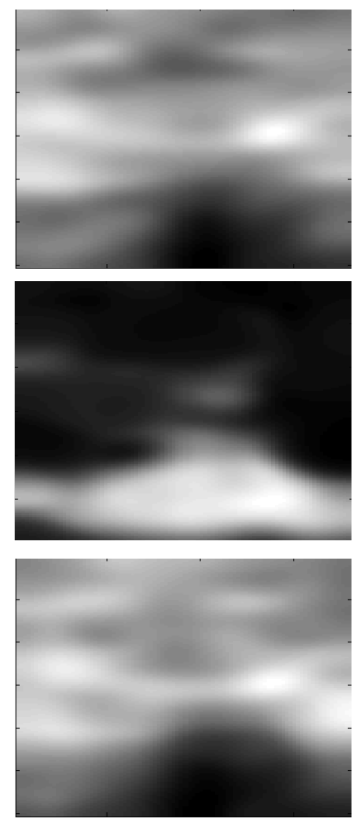

(b)
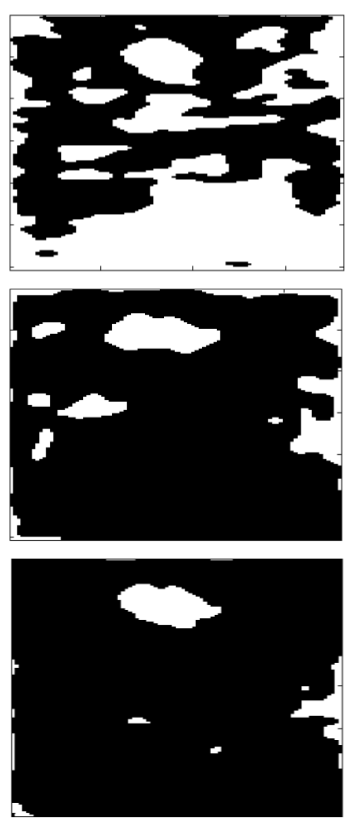

(c)
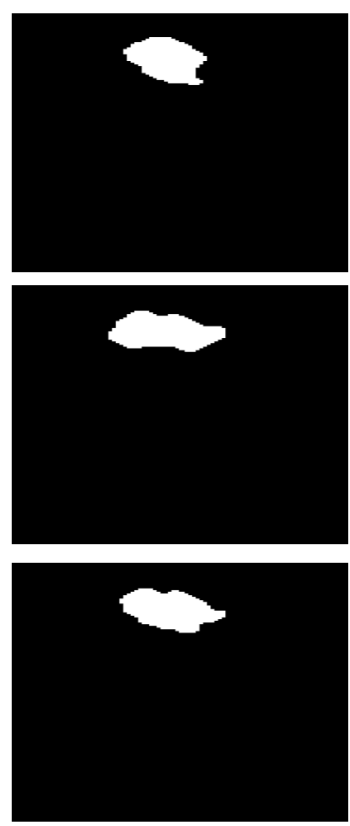

(d)

Figure 4. Segmentation results for Patient 2: using B-mode information alone (first row), using elastography information alone (second row) and combining both modalities (third row). Column (a) plots the original image, (b) the estimated bias field, (c) the first segmentation result and (d) the final refined segmentation.

\section{REFERENCES}

[1] Garra, B. S., Cespedes, E. I., Ophir, J., Spratt, S. R., Zuurbier, R. A., Magnant, C. M., and Pennanen, M. F., "Elastography of breast lesions: initial clinical results," Radiology 202(1), 79-86 (1997).

[2] Itoh, A., Ueno, E., Tohno, E., Kamma, H., Takahashi, H., Shiina, T., Yamakawa, M., and Matsumura, T., "Breast disease: clinical application of US elastography for diagnosis.," Radiology 239, 341-350 (May 2006).

[3] Zhi, H., Ou, B., Luo, B.-M., Feng, X., Wen, Y.-L., and Yang, H.-Y.. "Comparison of Ultrasound Elastography, Mammography, and Sonography in the Diagnosis of Solid Breast Lesions," J Ultrasound Med 26, 807-815 (June 2007).

[4] von Lavante, E. and Noble, J., "Segmentation of breast cancer masses in ultrasound using radio-frequency signal derived parameters and strain estimates," in [Biomedical Imaging: From Nano to Macro, 2008. ISBI 2008. 5th IEEE International Symposium on], 536 -539 (14-17 2008).

[5] Chang, R.-F., Chen, D.-R., Moon, W. K., and Lai, W.-R., "3-d ultrasound strain images for breast cancer diagnosis," International Congress Series 1281, 1069 - 1074 (2005). CARS 2005: Computer Assisted Radiology and Surgery.

[6] Xiao, G., Brady, M., Noble, J., and Zhang, Y., "Segmentation of ultrasound b-mode images with intensity inhomogeneity correction," Medical Imaging, IEEE Transactions on 21, 48 -57 (Jan. 2002).

[7] Besag, J., "Spatial interaction and the statistical analysis of lattice systems (with discussion)," Journal of the Royal Statistical Society 36(2), 192 - 326 (1974).

[8] Besag, J., "On the statistical analysis of dirty pictures," Journal of the Royal Statistical Society B-48, 259-302 (1986). 